\title{
ON CENTRAL AUTOMORPHISMS OF FINITE-BY-NILPOTENT GROUPS
}

\author{
by SILVANA FRANCIOSI and FRANCESCO DE GIOVANNI
}

(Received 24th October 1988)

\begin{abstract}
The effect of imposing a certain finiteness condition on the group of central automorphisms of a finite-by-nilpotent group is investigated. In particular it is shown that, if each central automorphism of a finite-by-nilpotent group $G$ has finite order, then the factor group $G / Z(G)$ has finite exponent. 1980 Mathematics subject classification (1985 Revision): 20F28.
\end{abstract}

\section{Introduction}

Many authors have investigated the structure of a group $G$ when a certain finiteness condition is imposed on the automorphism group Aut $G$ of $G$. In particular Robinson $([10,12])$ showed that, if the automorphism group Aut $G$ is Cernikov, then it is finite and the set of all elements of finite order of $G$ is a finite subgroup. Also Pettet [7] studied groups whose automorphism groups are locally finite and satisfy the minimal condition on primary subgroups. The imposition of finiteness conditions on the ranks of the automorphism groups was considered by Robinson and the authors in [3].

More recently there has been increasing interest in the structure of a group $G$ for which the group of central automorphisms Aut $_{C} G$ satisfies some finiteness condition. Clearly, dealing with this type of problem, one has to require some nilpotency condition on the group $G$, since when the centre $Z(G)$ is small, Aut $_{C} G$ is also small, but almost nothing can be said on the structure of $G$. In [2] finite-by-nilpotent groups $G$ for which Aut $_{C} G$ is countable were studied, while nilpotent groups whose groups of central automorphisms have finite exponent were investigated in [1].

Our main object here is to consider the behaviour of finite-by-nilpotent groups for which the groups of central automorphisms are periodic.

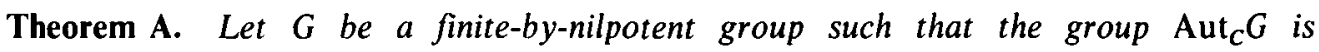
periodic. Then the factor group $G / Z(G)$ has finite exponent.

In the situation of Theorem A, the torsion subgroup of $G$ can be of infinite exponent. In fact there exists an abelian group whose torsion subgroup has infinite exponent and such that the automorphism group is a countable locally finite group of infinite exponent (see [15]). However, when the group $G$ is periodic, Theorem $\mathrm{A}$ has the following easy consequence (see also [1]). 
Corollary. Let $G$ be a periodic finite-by-nilpotent group such that the group Aut $_{C} G$ is periodic. Then $G$ has finite exponent.

The consideration of an extraspecial infinite $p$-group shows that in the situation of the Corollary the group $G$ can be infinite. In Section 2 some weaker results will be proved for groups which are either Černikov-by-nilpotent or hypercentral. However, Theorem A and Corollary become false for these groups, as the locally dihedral 2-group shows.

There are stronger results for finite-by-nilpotent groups whose groups of central automorphisms satisfy the minimal condition on primary subgroups.

Theorem B. Let $G$ be a finite-by-nilpotent group such that the group Aut $_{C} G$ is periodic.

(a) If Aut ${ }_{\mathrm{C}} \mathrm{G}$ satisfies the condition Min-p for a prime $p$, then the Sylow p-subgroups of $G$ are finite.

(b) If $\mathrm{Aut}_{C} G$ satisfies the condition Min-p for every prime $p$, then Aut $G$ is finite, and hence the elements of finite order of $G$ form a finite subgroup.

In particular Theorem B shows that, if $G$ is a finite-by-nilpotent group such that Aut $_{C} G$ is Cernikov, then Aut $G$ is finite. This is even true if $\mathrm{Aut}_{C} G$ satisfies the minimal condition on abelian subgroups, as will follow from the proof of Theorem B. In Section 3 some other results on groups $G$ such that $A_{u t} G$ is Černikov are proved. In particular there is constructed a soluble group $G$ such that $A t_{C} G$ is an infinite abelian group satisfying the minimal condition. Finally, in Section 4 we consider groups $G$ for which Aut $_{C} G$ satisfies the maximal condition on abelian subgroups.

We shall make frequent use of homological techniques. For these we refer to [14].

Notation. Our notation is standard and can be found in [8]. In particular we note:

$G_{a b}$ is the commutator factor group $G / G^{\prime}$.

$M(G)$ is the Schur multiplicator of the group $G$.

$\pi(G)$ is the set of prime divisors of the orders of elements of $G$.

If $\pi$ is a set of primes and $G$ is a locally nilpotent group, $G_{\pi}$ is the subgroup of all elements of $G$ whose order is a $\pi$-number.

tor $G$ is the set of all elements of finite order of the group $G$.

If $p$ is a prime and $A$ is an abelian group, $A[p]$ is the set of elements of order $p$ of $A$.

\section{Proof of Theorem A}

Our first lemma gives some information on the Schur multiplicator of certain Cernikov-by-nilpotent groups.

Lemma 2.1. Let $Q$ be a Černikov-by-nilpotent group which has no $p^{\infty}$-quotients for a prime $p$. Then the Schur multiplicator $M(Q)$ of $Q$ has no $p^{\infty}$-quotients. 
Proof. Since $Q$ is Cernikov-by-nilpotent, the last term $N$ of the lower central series of $Q$ is a Cernikov group and the factor group $Q / N$ is nilpotent. Let $R$ be the finite residual of $N$. As $R /[R, Q]$ is contained in the centre of $Q /[R, Q]$ and $Q / R$ is finite-bynilpotent, it follows that also $Q /[R, Q]$ is finite-by-nilpotent (see [8, Part 1, p. 113-117]). Hence $N /[R, Q]$ is finite, and so $R=[R, Q]$. In particular $H_{0}\left(Q / N, R N^{\prime} / N^{\prime}\right)=0$, and it follows from a result of Robinson (see [9, Theorem C]) that $H_{1}\left(Q / N, R N^{\prime} / N^{\prime}\right)$ has finite exponent. Since obviously $H_{1}\left(Q / N, N / R N^{\prime}\right)$ has finite exponent, the long exact homology sequence applied to the sequence of $Q / N$-modules

$$
R N^{\prime} / N^{\prime} \nrightarrow N_{a b} \rightarrow N / R N^{\prime}
$$

shows that $H_{1}\left(Q / N, N_{a b}\right)$ has finite exponent. Moreover it is well-known that the Schur multiplicator $M(N)$ of the Cernikov group $N$ is finite (see [11]), and hence by the Lyndon-Hochschild-Serre spectral sequence for homology it suffices to prove that the Schur multiplicator of the nilpotent group $Q / N$ has no $p^{\infty}$-quotients. Therefore we may suppose that $Q$ is nilpotent.

The abelian group $Q_{a b}$ has no $p^{\infty}$-sections, and hence it has finite torsion-free rank and its $p$-sections have finite exponent. Thus $Q_{a b}$ contains a finitely generated subgroup $L$ such that $Q_{a b} / L$ is the direct product of a group of finite exponent and a periodic group without elements of order $p$. This property is inherited by tensor products and homomorphic images, so that in particular the Schur multiplicator $M\left(Q_{a b}\right)$ has the same structure, and this proves the lemma when $Q$ is abelian. Suppose that $Q$ is not abelian, and let $K$ be the last non-trivial term of the lower central series of. $Q$. Then $K$ is a homomorphic image of the tensor product of finitely many copies of $Q_{a b}$. Application of Ganea's theorem to the central extension $K \succ Q \rightarrow Q / K$ gives an exact sequence

$$
K \otimes Q_{a b} \rightarrow M(Q) \rightarrow M(Q / K)
$$

(see $\left[14\right.$, p. 105]). Since by induction the group $M(Q / K)$ has no $p^{\infty}$-quotients, it follows that $M(Q)$ has no $p^{\infty}$-quotients.

Our next result gives information on the centre of a group $G$ with $\mathrm{Aut}_{C} G$ periodic. It can be proved even for Černikov-by-nilpotent groups.

Proposition 2.2. Let $G$ be a Cernikov-by-nilpotent group such that $\mathrm{Aut}_{C} G$ is periodic. Then the centre $Z(G)$ is reduced.

Proof. Assume that $Z(G)$ contains a subgroup $P$ of type $p^{\infty}$, and put $Q=G / P$. The homomorphism group $\operatorname{Hom}\left(Q_{a b}, P\right)$ is isomorphic with a subgroup of $A_{u t} G$, and so is periodic. Hence $Q$ has no $p^{\infty}$-quotients, and by Lemma 2.1 also the Schur multiplicator $M(Q)$ has no $p^{\infty}$-quotients. Then $\operatorname{Hom}(M(Q), P)$ is periodic. By the Universal Coefficients Theorem we have that

$$
H^{2}(Q, P) \simeq \operatorname{Ext}\left(Q_{a b}, P\right) \oplus \operatorname{Hom}(M(Q), P) \simeq \operatorname{Hom}(M(Q), P),
$$

and hence $H^{2}(Q, P)$ is periodic. Let $e$ be the order of the cohomology class $\Delta$ of the 
central extension $P>G \rightarrow Q$. If $\alpha$ is a $p$-adic integer such that $\alpha \equiv 0(\bmod p)$ (and $\alpha \equiv 0(\bmod 4)$ if $p=2)$, the position

$$
x^{\tau}=x^{1+e a}(x \in P)
$$

defines an automorphism of infinite order $\tau$ of $P$, and $\Delta \tau_{*}=\Delta$. Then $\tau$ can be extended to an automorphism $\bar{\tau}$ of $G$ acting trivially on $Q$; in particular $\bar{\tau}$ is a central automorphism of infinite order of $G$. This contradiction shows that $Z(G)$ has no subgroups of type $p^{\infty}$.

Assume now that $Z(G)$ contains a subgroup $V$ isomorphic with the additive group of rational numbers, and let $N$ be a Cernikov normal subgroup of $G$ with nilpotent factor group $G / N$. The group $\operatorname{Hom}\left(G / G^{\prime} V, V\right)$ is periodic, since it is isomorphic with a subgroup of $\operatorname{Aut}_{C} G$, and hence $G / V$ has no torsion-free abelian factors. Thus the nilpotent group $G / N V$ is periodic, and so $G / V$ is periodic. It follows that also the Schur multiplicator $M(G / V)$ of $G / V$ is periodic. Application of the Universal Coefficients Theorem gives that $H^{2}(G / V, V)=0$, and so $V$ is a direct factor of $G$. Therefore the non-periodic group Aut $V$ is isomorphic with a subgroup of $A_{u t} G$. This contradiction completes the proof.

Proof of Theorem A. Write $C=Z(G)$ and $Q=G / C$. It follows from Proposition 2.2 that $C$ is reduced, and hence each factor of the upper central series of $Q$ is also reduced (see [8 Part 2, p. 134]). Moreover the centre $Z(Q)$ is periodic, since it is isomorphic with a subgroup of Aut $_{c} G$.

Suppose that $Z_{i}(Q)$ is periodic for some integer $i \geqq 1$. Let $p$ be any prime such that the $p$-component of the abelian group $Z_{i}(Q) / Z_{i-1}(Q)$ has infinite exponent; then it is well-known that also the $p$-component of $C$ has infinite exponent (see [8 Part 1, p. 52]). Since the group $\operatorname{Hom}\left(Q_{a b}, C\right)$ is periodic, it follows that $Q_{a b}$ has no quotients which are the direct product of cyclic $p$-groups of unbounded orders. Every basic subgroup of an abelian p-group is an endomorphic image (see [4, Vol. 1, p. 152]), and hence the group $Q_{a b}$ has no quotients which are reduced $p$-groups of infinite exponent. Therefore every homomorphism from $Q_{a b}$ into the $p$-component of the reduced group $Z_{i}(Q) / Z_{i-1}(Q)$ has finite order.

Let $\phi$ be any homomorphism from $Q$ into $Z_{i}(Q) / Z_{i-1}(Q)$. Then $Q / \operatorname{ker} \phi$ is a periodic abelian group whose primary components have finite exponent. The set of primes $\pi(Q / \operatorname{ker} \phi)$ is contained in $\pi\left(Z_{i}(Q) / Z_{i-1}(Q)\right)$ and so also in $\pi(C)$. But $\operatorname{Hom}\left(Q_{a b}, C\right)$ is periodic, and this forces $\pi(Q / \operatorname{ker} \phi)$ to be finite. Therefore $Q / \operatorname{ker} \phi$ has finite exponent, and hence the homomorphism group $\operatorname{Hom}\left(Q_{a b}, Z_{i}(Q) / Z_{i-1}(Q)\right)$ is periodic. Since $Z_{i+1}(Q) / Z_{i}(Q)$ can be embedded in $\operatorname{Hom}\left(Q_{a b}, Z_{i}(Q) / Z_{i-1}(Q)\right)$, it follows that $Z_{i+1}(Q)$ is periodic. Thus the finite-by-nilpotent group $Q$ is periodic.

Let $N$ be a finite normal subgroup of $Q$ with nilpotent factor group $Q / N$, and assume that the Sylow $p$-subgroup $P$ of $Q / N$ has infinite exponent, for some prime $p$. Then clearly also the p-component of $C$ has infinite exponent. Let $B$ be a basic subgroup of the abelian $p$-group $P_{a b}$. Then $B$ is a homomorphic image of $P_{a b}$ and hence of $Q$, so that $\operatorname{Hom}(B, C)$ is isomorphic with a subgroup of $\operatorname{Aut}_{C} G$, and so is periodic. Since $B$ is the 
direct product of cyclic $p$-groups, it follows that $B$ has finite exponent, and hence $P_{a b}=B \times L$, where $L$ is radicable. Thus $P^{\prime}$ has finite exponent (see [8, Part 1, p. 55]). As $P$ is reduced, $P_{a b}$ is also reduced and so has finite exponent. This contradiction shows that every Sylow subgroup of $Q$ has finite exponent.

Since $\operatorname{Hom}\left(Q / N Q^{\prime}, C\right)$ is periodic, the set $\pi(Q) \cap \pi(C)$ is finite. But $\pi(Q) \backslash \pi(C)$ is also finite, as $G$ is finite-by-nilpotent. Therefore $\pi(Q)$ is finite and $Q$ has finite exponent.

It should be noted that, if $G$ is either hypercentral or Cernikov-by-nilpotent and Aut $_{C} G$ is periodic, the central factor group $G / Z(G)$ can be even non-periodic. In fact, let $\alpha \neq 1$ be a $p$-adic integer such that $\alpha \equiv 1(\bmod p)(\operatorname{and} \alpha \equiv 1(\bmod 4)$ if $p=2)$, and put $G=\langle\alpha\rangle \propto \mathbb{Z}\left(p^{\infty}\right)$. Then $G$ is a hypercentral Cernikov-by-abelian group and $Z(G)$ is finite, so that also $\operatorname{Aut}_{C} G$ is finite, but $G / Z(G)$ is not periodic. Note also that the automorphism group Aut $G$ is not periodic.

Proof of the Corollary. Let $C=Z(G)$ and $Q=G / C$. From Theorem A it follows that $Q$ has finite exponent and so, by the Universal Coefficients Theorem, $H^{2}(Q, C)$ also has finite exponent $e$. Assume that the $p$-component $C_{p}$ of $C$ has infinite exponent for some prime $p$. If $\alpha \neq 0$ is a $p$-adic integer such that $\alpha \equiv 0(\bmod p)($ and $\alpha \equiv 0(\bmod 4)$ if $p=2)$, the position

$$
x^{2}=x^{1+e \alpha}, \quad y^{z}=y \quad\left(x \in C_{p}, y \in C_{p^{\prime}}\right)
$$

defines an automorphism $\tau$ of $C$ of infinite order which can be extended to a central automorphism $\bar{\tau}$ of $G$. This contradiction shows that every primary component of $C$ has finite exponent.

If $\pi=\pi(C) \backslash \pi(Q)$, we have that $G=C_{\pi} \times K$. Then Aut $C_{\pi}$ is periodic, and hence $\pi$ must be finite. Therefore also $\pi(C)$ is finite, and $G$ has finite exponent.

If the condition of being periodic is imposed on the full automorphism group Aut $G$, then all these results can be stated for Cernikov-by-nilpotent groups. In fact we have the following.

Proposition 2.3. Let $G$ be a Cernikov-by-nilpotent group such that the group Aut $G$ is peiodic. Then $G$ is finite-by-nilpotent.

Proof. Let $N$ be a Černikov normal subgroup of $G$ with nilpotent factor group $G / N$, and let $R$ be the finite residual of $N$. Assume that $R \neq 1$, and let $p$ be a prime such that the p-component $P$ of $R$ is not trivial. By Proposition 2.2 the centre $C=Z(G)$ is reduced and so $P \cap C$ is finite. Therefore $H^{0}(G / R, P)$ is finite, and hence $H^{2}(G / R, P)$ has finite exponent (see [6]). Clearly $H^{1}(R / P, P)=H^{2}(R / P, P)=0$, and so from the LyndonHochschild-Serre spectral sequence for cohomology it follows that the group $H^{2}(G / P, P)$ has finite exponent. Let $e$ be the order of the cohomology class $\Delta$ of the extension $P>G \rightarrow G / P$, and let $\alpha \neq 0$ be a $p$-adic integer such that $\alpha \equiv 0(\bmod p)($ and $\alpha \equiv 0(\bmod 4)$ if $p=2$ ). The position

$$
x^{x}=x^{1+e x} \quad(x \in P)
$$


defines an automorphism of infinite order $\tau$ of $P$, and $\Delta \tau_{*}=\Delta$. Therefore $\tau$ can be extended to an automorphism of infinite order $\bar{\tau}$ of $G$, which acts trivially on $G / P$. This contradiction shows that $R=1$ and $G$ is finite-by-nilpotent.

In [1], Theorem D, an example is given of a hypercentral $p$-group $G$ such that Aut $_{C} G$ has exponent $p$ and $Z(G)$ is a reduced group of infinite exponent. In the following we will construct a hypercentral 2-group $G$ such that Aut $_{c} G$ has exponent 2 and $Z(G)$ is not reduced. In particular this shows that Proposition 2.2 does not hold for hypercentral groups.

Proposition 2.4. There exists a hypercentral 2-group $G$ such that Aut ${ }_{c} G$ has exponent 2 , but $Z(G)$ is not reduced.

Proof. Let $C=\left\langle c_{n} \mid n \in \mathbb{N}_{0}, \quad c_{0}=1, \quad c_{n+1}^{2}=c_{n}\right\rangle$ be a group of type $2^{\infty}$, and let $Q=D r_{n \in \mathbb{N}}\left(\left\langle\bar{u}_{n}\right\rangle \times\left\langle\bar{v}_{n}\right\rangle\right)$, where $\bar{u}_{n}$ and $\bar{v}_{n}$ have order $2^{n}$ for all $n$. If $C$ is considered as a trivial $Q$-module, from the Universal Coefficients Theorem it follows that $H^{2}(Q, C) \simeq$ $\operatorname{Hom}(M(Q), C)$. Since $M(Q)$ is the exterior square $Q \wedge Q$, the positions

$$
f\left(\bar{u}_{n}, \bar{v}_{n}\right)=c_{n}, f\left(\bar{u}_{i}, \bar{u}_{j}\right)=f\left(\bar{v}_{i}, \bar{v}_{j}\right)=f\left(\bar{u}_{i}, \bar{v}_{j}\right)=1 \quad \text { if } \quad i \neq j
$$

define an epimorphism $f: M(Q) \rightarrow C$, and so a central extension

$$
C>G_{0} \rightarrow Q
$$

Clearly we have that $C=G_{0}^{\prime}=Z\left(G_{0}\right)$.

If $\bar{x}$ is the inversion on $Q$, then $\bar{x}$ acts trivially on $Q \otimes Q$ and so also on $M(Q)$. Therefore $\bar{x}$ is induced by an automorphism $x$ of $G_{0}$ acting as the identity on $C$. Then $x^{2}=1$ and so the semidirect product $G=\langle x\rangle \ltimes G_{0}$ is a hypercentral 2-group such that $C=Z(G)$. For each $n \in \mathbb{N}$ let $u_{n}$ and $v_{n}$ be elements of $G_{0}$ such that $u_{n} C=\bar{u}_{n}$ and $v_{n} C=\bar{v}_{n}$. Then $G^{\prime}=\left\langle u_{n}^{2}, v_{n}^{2} \mid n \in \mathbb{N}\right\rangle \geqq C$ and so $G / G^{\prime}$ has exponent 2 . The group Aut ${ }_{C} G$ acts trivially on $G^{\prime}$ and so also on $C$; thus $\operatorname{Aut}_{C} G \simeq \operatorname{Hom}\left(G_{a b}, C\right)$ is an infinite elementary abelian 2-group.

Remark. If $G$ is a hypercentral group such that $A u_{C} G$ is periodic, then $Z(G)$ does not contain any copy of the additive group of rational numbers. In fact, assume that $C=Z(G)$ contains a subgroup $A$ isomorphic with $\mathbb{Q}(+)$. Since $\operatorname{Hom}\left(G / G^{\prime} C, C\right)$ is periodic, the group $G / G^{\prime} C$ has no torsion-free quotients and so is periodic. Then the hypercentral group $G / C$ is periodic (see [13]) and so also $G^{\prime}$ is periodic. Clearly $G_{a b}=A G^{\prime} / G^{\prime} \times K / G^{\prime}$ and so $G=A K=A \times K$, as $A \cap K=A \cap G^{\prime}=1$. It follows that the non-periodic group Aut $A$ can be embedded in $A_{u t} G$. This contradiction proves that $C$ does not contain copies of $\mathbb{Q}(+)$.

\section{Proof of Theorem B}

The next lemma is the main tool in the proof of Theorem $B(b)$. 
Lemma 3.1. Let $G$ be a group and let $C=Z(G), Q=G / C, T=\operatorname{tor} C$ and $\bar{C}=C / T$. If the group $\Lambda$ of all automorphisms of $\bar{C}$ induced by automorphisms of $G$ acting trivially on $Q$ and $T$ is periodic, then $\Lambda$ has finite exponent dividing 12. Moreover every involution of $\Lambda$ is central, and so $\Lambda$ is locally finite.

Proof. Let $\theta$ be any element of $\Lambda$ and let $\tau$ be an automorphism of $G$ acting trivially on $Q$ and $T$ and inducing $\theta$ on $\bar{C}$. Write $\bar{\tau}$ for the automorphism induced by $\tau$ on $C$. Suppose first that $\theta$ has order $n=p^{k}>3$, where $p$ is an odd prime. If

$$
\phi=1-\theta+\theta^{2}-\theta^{3}+\cdots+\theta^{n-3} \text { and } \psi=1-\bar{\tau}+\bar{\tau}^{2}-\bar{\tau}^{3}+\cdots+\bar{\tau}^{n-3},
$$

then $\phi$ is an automorphism of $\bar{C}$, its inverse being $\theta^{2}-\theta^{4}+\theta^{6}-\cdots+\theta^{n-1}$ if $n \equiv 1(\bmod 4)$ and $\theta^{3}-\theta^{5}+\theta^{7}-\cdots+\theta^{n}$ if $n \equiv-1(\bmod 4)$. Moreover $\bar{\tau}$ acts trivially on $T$ and so $\psi$ acts trivially on $T$. Since $\psi$ induces $\phi$ on $\bar{C}$, it follows that $\psi$ is an automorphism of $C$. If $\Delta$ is the cohomology class of the central extension $C \nrightarrow G \rightarrow Q$, we have that $\Delta \bar{\tau}_{*}=\Delta$ and so also $\Delta \psi_{*}=\Delta$. Therefore $\psi$ can be extended to an automorphism $\xi$ of $G$ acting trivially on $Q$ and $T$. But $\xi$ acts on $\bar{C}$ as $\phi$, and so $\phi$ belongs to $\Lambda$. This is impossible as $\phi$ has infinite order (see [4, Vol. 2, p. 268]). Suppose now that $\theta$ has order 8 . If

$$
\phi=1+\left(1-\theta^{4}\right)\left(1+\theta-\theta^{3}\right) \text { and } \psi=1+\left(1-\bar{\tau}^{4}\right)\left(1+\bar{\tau}-\bar{\tau}^{3}\right)
$$

then $\phi$ is an automorphism of $\bar{C}$, its inverse being $1+\left(1-\theta^{4}\right)\left(1-\theta+\theta^{3}\right)$. As above we get a contradiction. Therefore $\Lambda$ has finite exponent dividing 12 .

Let $\alpha$ and $\beta$ be elements of $\Lambda$ such that $\alpha^{2}=1$. Then there exist automorphisms $\gamma$ and $\delta$ of $G$, acting trivially on $Q$ and $T$, which induce $\alpha$ and $\beta$, respectively. Consider the restrictions $\bar{\gamma}$ and $\bar{\delta}$ of $\gamma$ and $\delta$ to $C$, and put

$$
\hat{\lambda}=(1+\alpha) \beta(1-\alpha), \mu=(1-\alpha) \beta(1+\alpha) \quad \text { and } \quad \bar{\lambda}=(1+\bar{\gamma}) \bar{\delta}(1-\bar{\gamma}), \bar{\mu}=(1-\bar{\gamma}) \bar{\delta}(1+\vec{\gamma})
$$

Thus $\lambda^{2}=\mu^{2}=0$, and so $1+\lambda$ and $1+\mu$ are automorphisms of $\bar{C}$. Since $1+\bar{\lambda}$ and $1+\bar{\mu}$ act trivially on $T$, it follows that they are automorphisms of $C$. But $\Delta \bar{\gamma}_{*}=\Delta \bar{\delta}_{*}=\Delta$ and so $\Delta \bar{\lambda}_{*}=\Delta \bar{\mu}_{*}=0$. This proves that $1+\bar{\lambda}$ and $1+\bar{\mu}$ can be extended to automorphisms of $G$ acting trivially on $Q$ and $T$. Therefore $1+\lambda$ and $1+\mu$ belong to $\Lambda$ and so there exists a positive integer $n$ such that $(1+\lambda)^{n}=(1+\mu)^{n}=1$. Since $\bar{C}$ is torsion-free and $\lambda^{2}=\mu^{2}=0$, it follows that $\lambda=\mu=0$. Hence $0=\lambda-\mu=2(\alpha \beta-\beta \alpha)$, so that $\alpha \beta=\beta \alpha$ and $\alpha \in Z(\Lambda)$. Thus $\Lambda / Z(\Lambda)$ has exponent dividing 6 , and then is locally finite by the positive solution of the Burnside problem for exponent 6 (see [5]). It follows that also $\Lambda$ is locally finite.

Proof of Theorem B. (a) Write $C=Z(G)$ and $Q=G / C$. The group $Q$ has finite exponent by Theorem $A$, and so also the commutator subgroup $G^{\prime}$ has finite exponent. Assume that the $p$-component $C_{p}$ of $C$ is infinite. Since $C$ is reduced by Proposition 2.2, the socle $S$ of $C_{p}$ is also infinite, and then $S=S_{1} \times S_{2}$, where $S_{1}$ and $S_{2}$ are both infinite. The homomorphism group $\operatorname{Hom}\left(G / G^{\prime} S_{1}, S_{1}\right)$ is isomorphic with a subgroup of $A^{4} c_{C} G$, and so satisfies Min-p. Therefore $G / G^{\prime} S_{1}$ has no quotients of order $p$.

Let $n$ be a positive integer such that $\gamma_{n}(G)$ is finite. Then the Sylow $p$-subgroup of the 
nilpotent group $G / \gamma_{n}(G) S_{1}$ is a non-trivial radicable group (see [8, Part 2, p. 125]). Since the finite-by-nilpotent group $G$ is reduced (see [8, Part 2, p. 134]) and $\gamma_{n}(G) S_{1}$ has finite exponent, it follows that $G / \gamma_{n}(G) S_{1}$ is also reduced. This contradiction shows that the p-component of $C$ is finite.

There exists a positive integer $m$ such that $G / Z_{m}(G)$ is finite (see [8, Part 1, p. 117]). If $C_{p}=1$, it follows that the Sylow p-subgroups of $G$ are finite, since $\pi\left(Z_{m}(G)\right) \subseteq \pi(C)$ (see [8, Part 1, p. 53]). Suppose now that $C_{p} \neq 1$. The abelian p-group Hom $\left(Q_{a b}, C_{p}\right)$ is finite, and so the $p$-component of $Q_{a b}$ is also finite. Hence the Sylow p-subgroups of $Q$ are finite, and this proves part (a) of Theorem B.

(b) From the proof of (a) it follows that $C$ and $Q$ have finite Sylow $p$-subgroups for every prime $p$. In particular $Q$ is finite, since it has finite exponent. Assume that the set of primes $\pi(C) \backslash \pi(Q)$ contains infinitely many elements $p_{n}(n \in \mathbb{N})$. Since every $C_{p_{i}}$ is a finite central subgroup of $G$, it follows easily that, for every positive integer $n$,

$$
G=C_{p_{1}} \times \cdots \times C_{p_{n}} \times K_{n},
$$

where $K_{1}>K_{2}>\cdots>K_{n}$. Let $\alpha_{n}$ be the central automorphism of $G$ acting trivially on $K_{n}$ and as the inversion on $C_{p_{1}} \times \cdots \times C_{p_{n}}$. Then $\left\langle\alpha_{n} \mid n \in \mathbb{N}\right\rangle$ is an infinite elementary abelian 2-group. This contradiction shows that $\pi(C)$ is finite and hence the torsion subgroup $T$ of $C$ is finite.

Write $\Gamma=$ Aut $_{C} G, \Gamma_{1}=C_{\Gamma}(T)$ and $\Gamma_{2}=C_{\Gamma_{1}}(C / T)$. Then clearly $\Gamma / \Gamma_{1}$ is finite, and from Lemma 3.1 it follows that $\Gamma_{1} / \Gamma_{2}$ is a locally finite group with finite exponent. If $\Gamma_{3}=C_{\Gamma_{2}}(C)$, then $\Gamma_{2} / \Gamma_{3}$ is isomorphic with a subgroup of $\operatorname{Hom}(C / T, T)$ and $\Gamma_{3}$ is isomorphic with a subgroup of $\operatorname{Hom}\left(G / G^{\prime} C, C\right)$. Therefore $\Gamma_{2} / \Gamma_{3}$ and $\Gamma_{3}$ are abelian groups of finite exponent. Then $A_{u t} G$ is a locally finite group with finite exponent, and so its abelian subgroups are finite. The Hall-Kulatilaka-Kargapolov theorem (see [8, Part 1, p. 95]) proves now that Aut ${ }_{C} G$ is finite. Since $\operatorname{Aut}_{C} G=\mathrm{C}_{\text {Aut }}(Q)$ and $Q$ is finite, it follows that $\operatorname{Aut} G$ is also finite.

The last part of this section concerns with groups for which the group of central automorphisms is Cernikov.

Proposition 3.2. Let $G$ be a periodic hypercentral group such that $\mathrm{Aut}_{C} G$ is a Cernikov group. Then $\mathrm{Aut}_{c} G$ is finite.

Proof. Since $G=\operatorname{Dr}_{p \in \pi(G)} G_{p}$, we have also that $\operatorname{Aut}_{C} G \simeq \mathrm{Cr}_{p \in \pi(G)} \mathrm{Aut}_{C} G_{p}$. But Aut ${ }_{C} G_{p}$ is non-trivial if $p$ is an odd prime in $\pi(G)$, and hence the set $\pi(G)$ is finite. Thus we may suppose that $G$ is a $p$-group. Write $C=Z(G)$ and $Q=G / C$. If $Q$ has no quotients of order $p$, then $Q_{a b}$ is radicable and so the hypercentral $p$-group $Q$ is abelian (see [8, Part 2, p. 125]). Therefore $G$ is nilpotent, and in this case the result follows from Theorem $\mathrm{B}(\mathrm{b})$. Thus we may suppose that $Q$ has a quotient of order $p$. As $G$ is periodic, the homomorphism group $\operatorname{Hom}\left(Q_{a b}, C\right)$ is reduced (see [4, Vol. 1, p. 193]) and so finite, since it is isomorphic with a subgroup of $A_{u t} G$. It follows that $C$ is a Cernikov group, and so $\operatorname{Aut}_{C} G / C_{\mathrm{Aut}_{C} G}(C)$ is finite (see $\left[8\right.$, Part 1, p. 85]). Moreover $C_{\mathrm{Aut}_{C} G}(C) \simeq$ $\operatorname{Hom}\left(Q_{a b}, C\right)$ is finite and so $\mathrm{Aut}_{c} G$ is also finite. 
Note that, if $G$ is a periodic hypercentral group such that $A_{u t} G$ is Cernikov, the automorphism group Aut $G$ can be infinite, as the consideration of the locally dihedral 2-group shows.

In [12] it was proved that an infinite Cernikov group cannot be the group of all automorphisms of a group. The following example shows that the situation is different for the group of central automorphisms.

Example. There exists a soluble group $G$ such that $\mathrm{Aut}_{C} G$ is an infinite abelian group satisfying the minimal condition.

Proof. Let $p$ be a prime and consider two groups $A$ and $B$ of type $p^{\infty}$ with generators $1=a_{0}, a_{1}, a_{2}, \ldots$ and $1=b_{0}, b_{1}, b_{2}, \ldots$, such that $a_{n+1}^{p}=a_{n}, b_{n+1}^{p}=b_{n}$. Let $\lambda$ be a $p$-adic unit with infinite multiplicative order, and consider the automorphisms $\alpha$ and $\beta$ of $H=A \times B$ defined by

$$
a_{n}^{\alpha}=a_{n}^{\lambda}, \quad a_{n}^{\beta}=a_{n} b_{n}, \quad b_{n}^{\alpha}=b_{n}^{\beta}=b_{n} \quad(n \in \mathbb{N}) .
$$

Write $K=\langle\alpha, \beta\rangle$ and $G=K \ltimes H$. Clearly $K$ acts trivially on $B$ and induces an infinite cyclic group of automorphisms on $H / B$, so that $K^{\prime}$ is abelian and $G^{(3)}=1$. We have that $B=[A, \beta]$ and $A=[A, \alpha]$, so that $H \leqq G^{\prime}$ and $G_{a b}$ is a finitely generated infinite abelian group. Since $Z(G)=B \leqq G^{\prime}$, the group $\operatorname{Aut}_{c} G$ is isomorphic with $\operatorname{Hom}\left(G_{a b}, B\right)$, and hence it is an infinite abelian gropup satisfying the minimal condition.

In the above example the centre $Z(G)$ of $G$ is a Cernikov group. Our next result shows in particular that this situation cannot occur for periodic groups.

Proposition 3.3. Let $G$ be a group such that Aut $_{C} G$ is a Črnikov group.

(a) If $G$ is periodic and $Z(G)$ satisfies the condition Min-p for every prime $p$, then Aut $_{C} G$ is finite.

(b) If $Z(G)$ is finite, then Aut $_{c} G$ is finite.

(c) If $G / Z(G)$ is finite, then the automorphism group Aut $G$ is finite.

Proof. Write $C=Z(G)$ and $Q=G / C$.

(a) For every prime $p$ the $p$-component $C_{p}$ of $C$ satisfies the minimal condition, and so the periodic group $\mathrm{Aut}_{c} G$ induces on $C_{p}$ a finite group of automorphisms (see [8, Part 1, p. 85]). Therefore the finite residual $\Theta$ of Aut $_{C} G$ acts trivially on $C$, and so is isomorphic with a subgroup of $\operatorname{Hom}\left(Q_{a b}, C\right)$. But $\operatorname{Hom}\left(Q_{a b}, C\right)$ is reduced (see $[4, \mathrm{Vol}$. 1, p. 193]) and hence $\Theta=1$. Therefore $A_{u t} G$ is finite.

(b) As $C$ is finite, the factor group $\operatorname{Aut}_{C} G / C_{\text {Aut } G^{G}}(C)$ is also finite. Moreover $C_{\text {Aut } C G(C)}$ is isomorphic with a subgroup of $\operatorname{Hom}\left(Q_{a b}, C\right)$ and so has finite exponent. Thus $C_{\text {Autc }}(C)$ is finite, and also $\mathrm{Aut}_{c} G$ is finite.

(c) Since Aut $_{C} G=C_{\text {Aut }}(Q)$, the factor group Aut $G / A_{A_{C}} G$ is finite. Hence Aut $G$ is a Cernikov group, and so is finite by a result of Robinson (see [12]). 


\section{The maximal condition on abelian subgroups}

As was already noted in Section 2, there exist artinian-by-nilpotent groups $G$ for which $\mathrm{Aut}_{c} G$ satisfies the minimal condition on abelian subgroups, but Aut $G$ is even non-periodic. In the dual situation, dealing with the maximal condition, the structure of Aut $_{C} G$ is inherited by Aut $G$.

Theorem 4.1. Let $G$ be a soluble-by-finite group which is also noetherian-by-nilpotent. If $\mathrm{Aut}_{C} G$ satisfies the maximal condition on abelian subgroups, then the automorphism group Aut $G$ satisfies the maximal condition on abelian subgroups, and the Sylow subgroups of $G$ are finite.

Proof. Write $C=Z(G)$ and $Q=G / C$. The centre $Z(Q)$ is isomorphic with an abelian subgroup of $\operatorname{Aut}_{C} G$, and so is finitely generated. Suppose that $Z_{i}(Q)$ is finitely generated for some positive integer $i$, and put $A_{i}=Z_{i}(Q) / Z_{i-1}(Q)$. If $p$ is a prime divisor of the order of the torsion subgroup $T_{i}$ of $A_{i}$, then $C$ contains elements of order $p$ (see [8, Part 1, p. 53]). Moreover the elementary abelian group $\operatorname{Hom}\left(Q_{a b}, C[p]\right)$ is isomorphic with a subgroup of $\operatorname{Aut}_{c} G$, and so is finite. Therefore $Q_{a b} /\left(Q_{a b}\right)^{p}$ is finite. It follows that $Q_{a b} /\left(Q_{a b}\right)^{e}$ is finite, where $e$ is the order of $T_{i}$, and hence $\operatorname{Hom}\left(Q_{a b}, T_{i}\right)$ is also finite.

Suppose that $\operatorname{Hom}\left(Q_{a b}, A_{i} / T_{i}\right) \neq 0$. Then $Q_{a b}$ has an infinite cyclic factor, and hence $C \simeq \operatorname{Hom}(\mathbb{Z}, C)$ is isomorphic with a subgroup of $\operatorname{Aut}_{C} G$, and so is finitely generated. Since $A_{i}$ is not periodic, it follows that also $C$ is not periodic, so that $\operatorname{Hom}\left(Q_{a b}, \mathbb{Z}\right)$ can be embedded in $\operatorname{Aut}_{c} G$. Thus $\operatorname{Hom}\left(Q_{a b}, \mathbb{Z}\right)$ is finitely generated. But $\operatorname{Hom}\left(Q_{a b}, A_{i} / T_{i}\right)$ is isomorphic with the direct sum of finitely many copies of $\operatorname{Hom}\left(Q_{a b}, \mathbb{Z}\right)$, and so is finitely generated. This shows that $\operatorname{Hom}\left(Q_{a b}, A_{i}\right)$ is finitely generated. As $Z_{i+1}(Q) / Z_{i}(Q)$ can be embedded in $\operatorname{Hom}\left(Q_{a b}, A_{i}\right)$, it follows that $Z_{i+1}(Q)$ is also finitely generated.

By hypothesis $Q$ is polycyclic-by-finite-by-nilpotent, and hence there exists a positive integer $n$ such that $Q / Z_{n}(Q)$ is polycyclic-by-finite (see [8, Part 1, p. 119]). Since $Z_{n}(Q)$ is finitely generated, it follows that $Q$ is polycyclic-by-finite. The factor group $A u t G / A u t_{C} G$ is isomorphic with a subgroup of Aut $\&$, and so its abelian subgroups are finitely generated (see [8, Part 1, p. 82]). Therefore Aut $G$ satisfies the maximal condition on abelian subgroups. Finally the Sylow subgroups of $G$ are finite by Theorem 1 (b) of [3].

In the hypotheses of Theorem 4.1, the group $G$ can have infinite periodic subgroups. In fact in [3, Theorem 3 (ii)], there was constructed a group $G$ such that Aut $G$ is polycyclic (and so in particular $G$ is polycyclic-by-abelian), but $G$ contains an infinite abelian periodic subgroup.

\section{REFERENCES}

1. M. Curzio, D. J. S. Robinson, H. Smith and J. Wiegold, Some remarks on central automorphisms of hypercentral groups, Arch. Math. (Basel), to appear.

2. S. Franciosi and F. DE GiovanNi, A note on groups with countable automorphism groups, Arch. Math. (Basel) 47 (1986), 12-16.

3. S. Franciosi, F. de Giovanni and D. J. S. Robinson, On torsion in groups whose automorphism groups have finite rank, Rocky Mountain J. Math. 17 (1987), 431-445. 
4. L. Fuchs, Infinite Abelian Groups (Academic Press, New York-London, 1970-73).

5. M. HALL JR., The Theory of Groups (MacMillan, New York, 1959).

6. J. C. Lennox and D. J. S. Robinson, Soluble products of nilpotent groups, Rend. Sem. Mat. Univ. Padova 62 (1980), 261-280.

7. M. R. Pettet, Locally finite groups as automorphism groups, Arch. Math. (Basel) 48 (1987), $1-9$.

8. D. J. S. Robinson, Finiteness Conditions and Generalized Soluble Groups (Springer, Berlin, 1972).

9. D. J. S. Robinson, The vanishing of certain homology and cohomology groups, J. Pure Appl. Algebra 7 (1976), 145-176.

10. D. J. S. Robinson, A contribution to the theory of groups with finitely many automorphisms, Proc. London Math. Soc. (3) 35 (1977), 34-54.

11. D. J. S. Robinson, Homology of group extensions with divisible abelian kernel, J. Pure Appl. Algebra 14 (1979), 145-165.

12. D. J. S. Robinson, Infinite torsion groups as automorphism groups, Quart. J. Math. Oxford (2) 30 (1979), 351-364.

13. D. J. S. Robinson, On the homology of hypercentral groups, Arch. Math. (Basel) 32 (1979), 223-226.

14. U. Stammвach, Homology in Group Theory (Lecture Notes in Mathematics 359, Springer, Berlin, 1973).

15. J. Zimmerman, Countable torsion FC-groups as automorphism groups, Arch. Math. (Basel) 43 (1984), 108-116.

Istituto di Matematica

FaColta di SCIENZE

Universitá di SALERNo

I-84100 SAlerno, Italy
Dipartimento di Matematica

Universitá di Napoli

Via Mezzocannone 8

I-80134 NAPOLI, ItALY 